\title{
The hawk-goose phenomenon: A replication and an extension'
}

MARSHA GREEN, RONALD GREEN AND W. J. CARR

TEMPLE UNIVERSITY

Mallard ducks, having no prior experience with objects passing overhead, were more active in the presence of the silhouette of a hawk than that of a goose. However, Ss were equally responsive to a triangle moving either base-forward or apex-forward. The results confirm earlier reports of the hawk-goose phenomenon, and cast doubt upon its explanation in terms of differences in the rate of change of visual stimulation produced by the two silhouettes.

Lorenz (1939) and Tinbergen (1948) reported that some gallinaceous birds exhibit escape reactions when the silhouette of a hawk is passed overhead, but not when the same silhouette is moved in the opposite direction, thus simulating the appearance of a goose. More recently, Melzack, Penick, \& Beckett(1959) reported the differential reaction in mallards, which had no prior experience with objects moving overhead. Other investigators (Hirsch, Lindley, \& Tolman, 1955; Rockett, 1955; McNiven, 1960) failed to confirm the findings in chickens and other species, but their techniques have been criticized by Tinbergen (1957) and Lorenz (1961)。 The present experiment was performed in an attempt to replicate the hawk-goose phenomenon in mallards, even though the birds had never seen objects moving overhead.

As a secondary purpose, the present investigation was also designed to test the hypothesis offered by Schneirla (1959, pp. 16-17) that the differential reaction exhibited by birds to the hawk and goose silhouettes stems not from the configurational properties of the silhouettes (e.g. short neck versus long neck, as suggested by Tinbergen, 1951, p. 77), but rather from the difference in the rate of change of visual stimulation produced by the two silhouettes as they pass overhead. Schneirla hypothesized that the blunt leading edge of the hawk pattern causes a more abrupt darkening of the visual field than does the relatively tapered leading edge of the goose pattern, and that the differential reaction of the Ss is produced by the resulting rapid versus gradual retinal changes. Such a consideration led Schneirla to suggest that a triangle moving base-forward or apex-forward might produce the same effects as do the hawk and goose, respectively.

Method

Nineteen mallard ducks (Anas platyrynchos) served as Ss. They were hatched in an incubator, kept in a brooder for 14 days, and then maintained in an indoor pen, the construction of which prevented them from seeing flying objects, other than insects. The Ss were fed an ad libitum diet of Purina duck mash or pellets and water. Testing occurred 44-47 days after hatching.

The amount of activity exhibited by $S$ was measured in response to each of four test patterns: the silhouette of a hawk, the silhouette of a goose (i.e. the hawk silhouette flying backwards), a triangle moving baseforward, and a triangle moving apex-forward. The Ss recieved one test per day for four consecutive days, and the order of presentation of the test patterns was randomized over successive tests. The Ss were tested individually; those birds not being tested were kept in an adjoining pen, from which they could not see the test patterns.

A rectangular pen, measuring 70 by 30 in., served both as the rearing and testing site. The test patterns were presented to $\mathrm{S}$ by passing them $6 \mathrm{ft}_{\text {. overhead, }}$ along the long axis of the pen. The patterns were attached to a guy wire and pulley arrangement, which was moved by hand at the approximate rate of $1 \mathrm{ft}$. per second. Movement of the patterns always began at the same end of the pen, and the patterns were out of S's view at the beginning and end of each test. The Es observed Ss from behind a screen.

The floor of the test pen was divided into seven blocks, each measuring 10 by 30 in., and a test began only when $S$ was standing quietly in one of the three center blocks. The dependent measure was the number of blocks $S$ entered during the first 15 sec. after the test pattern came into view. A similar measure was used by Kratzig (1940), Hirsch et al (1955), and by Melsack et al (1959).

The test patterns used in the present experiment were made from heavy cardboard and painted flat black. The hawk-goose silhouette was closely patterned after one of several used by Tinbergen $(1951$, p. 78). Its wing span was $8 \mathrm{in}$. and the body measured 6 in. in length. The base and altitude of the triangle were $8 \mathrm{in}$. and 4 in. respectively. Since the area of the triangle exceeded that of the hawk-goose silhouette by a ratio of 1.5 to unity, the triangle could be expected to produce a greater change in visual stimulation, as it passed overhead, than did the hawk-goose silhouette. The ceiling of the room was painted flat gray, in order to provide reasonable contrast with the test patterns.

Results and Discussion

Table 1 shows the mean and standard error of the ranked activity scores exhibited by $S$ in the presence of each of the four test patterns. Differences in activity scores attributable to treatments (i.e. test patterns) were statistically significant, as indicated by an analysis of variance of ranked data $\left(X^{2}\right.$ ranks $=10.2, d f=3$, 
Table 1. Means and Standard Errors of the Ranked Activity Scores in Response to the Four Test Pattems

\begin{tabular}{lcccc} 
& $\begin{array}{c}\text { Hawk } \\
\text { Pattern }\end{array}$ & $\begin{array}{c}\text { Goose } \\
\text { Pattern }\end{array}$ & $\begin{array}{c}\text { Test Pattern } \\
\text { Triangle } \\
\text { Base Forward Apex Forward }\end{array}$ & $\begin{array}{c}\text { Triangle } \\
\text { Ape }\end{array}$ \\
\hline Mean Rank & 3.53 & 2.37 & 1.92 & 2.18 \\
S. E. & 0.16 & 0.18 & 0.14 & 0.16 \\
\hline
\end{tabular}

Note. A high mean rank indicates a high level of activity.

$\mathrm{p}<.02)$. Moreover, the results of a Newman-Keuls test showed that the activity scores in response to the hawk pattern were significantly greater $(p<.01)$ than those elicited by the other patterns, but observed differences in activity scores produced by the other three patterns were not significant.

The results of the present experiment confirm earlier reports that mallard ducks are more active when the silhouette of a hawk is passed overhead than they are when the same silhouette is moved in the opposite direction, thus simulating the appearance of a goose. The high level of activity produced by the hawk silhouette has been linked to the mallard's escape pattern (Kratzig, 1940; Tinbergen, 1957; and Melzack et al, 1959). This differential responsiveness to the hawk-goose silhouette is the more remarkable because the birds had no prior experience with objects moving overhead.

On the other hand, no significant difference was observed in the amount of activity exhibited by Ss in the presence of the triangle moving base-forward, as compared with the same pattern moving apex-forward. Therefore, since both triangles elicited about the same low level of activity as did the goose silhouette, it would appear that the birds were responding to a configurational property present in the hawk silhouette and absent in the goose silhouette and the triangles.
Tinbergen (1951, pp. 30-31 and 77-78) has suggested that the short neck of the hawk constitutes a signstimulus which releases the escape pattern in the mallards. However, the identification of the critical aspect of the hawk silhouette awaits the results of additional research.

\section{References}

Hirsch, J., Lindley, R. H., \& Tolman, E. C. An experimental test of an alleged innate sign stimulus. J. comp. physiol. Psychol. $1955,48,278-280$

Kratzig, H. Untersuchungen zur Lebensweise des Moorschneehuhns Lagopus l. lagopus, wahrend der Jugendentwicklung. J. Ornithol., $1940,88,139-163$.

Lorenz, K. Z. Vergleichende Verhaltensforschung. Zool. Anz. Suppl. , 1939, 12, 69-102.

Lorenz, K. Z, Phylogenetische Anpassung und adaptive Modifikation des Verhaltens. Z. Tierpsychol., 1961, 18, 139-187.

McNiven, $\mathbf{H}$. "Social-releaser mechanisms" in birds-a controlled replication of Tinbergen's study. Psychol. Rec., 1960, 10 , 259-265.

Melzack, R., Penick, E., \& Beckett, A. The problem of "innate fear" of the hawk shape: an experimental study with mallard ducks. J. comp. physiol. Psychol., 1959, 52, 694-698.

Rockett, P. C. A note on "an experimental test of an alleged innate sign stimulus" by Hirsch, Lindley, and Tolman. Percept. mot. Skills, 1955, 5, 155-156.

Schneirla, T. C. An evolutionary and developmental theory of biphasic processes underlying approach and withdrawal. In $\mathrm{M}$. R. Jones (Ed.), Nebraska symposium on motivation. Lincoln, Neb.: University of Nebraska Press, 1959. Pp. 1-42.

Tinbergen, N. Social releasers and the experimental method required for their study. Wilson Bull., 1948, 60, 6-51.

Tinbergen, $\mathbf{N}$. The study of instinct. New York: Oxford University Press, 1951.

Tinbergen, $\mathbf{N}$. On anti-predator responses in certain birds-a reply. J. comp. physiol. Psychol., 1957, 50, 412-414.

\section{Nole}

1. This investigation was supported in part by a research grant from the T. L. Bolton Trust Fund. Temple University. The senior author is the recipient of a National Science Foundation Cooperative Fellow ship. 